\title{
Mobbing o acoso laboral. Revisión del tema en Colombia*
}

\author{
Mobbing or workplace harassment. Review of the issue in Colombia \\ Mobbing ou harcèlement au travail. Révision du sujet en Colombie
}

José G. Carvajal Orozco

Profesor Asociado, Coordinador del Grupo de Investigación en

Filosofía y Teoría en Administración - Categoría D,

Universidad Nacional, Manizales - Colombia.

MSc en Ciencias de la Organización de la Universidad del Valle (2006).

E-mail: jgcarvajalo@unal.edu.co

Carlos A. Dávila Londoño Investigador del Grupo de Investigación en Filosofía y Teoría en Administración - Categoría D,

Universidad Nacional, Manizales - Colombia. Administrador de Empresas Universidad Nacional Sede Manizales. Maestría en Administración (c), perfil investigativo de la Universidad Nacional Sede Manizales.

E-mail: cadavilal@unal.edu.co

Revisión de tema según clasificación COLCIENCIAS Recepción: 15/02/2013 Corrección: 10/04/2013 Aprobación: 02/05/2013

\section{Resumen}

El mobbing se reconoce como una línea de trabajo promisoria y de alto impacto sobre el hombre, las organizaciones y la sociedad. En Latinoamérica y en específico en Colombia, se identifica poco desarrollo de la temática, lo que constituye una debilidad frente a las posibilidades de conocer e intervenir sobre ésta, que se acepta como una realidad creciente en el mundo. El objetivo de este artículo es presentar los resultados de la revisión y el análisis del tema del mobbing para Colombia. El documento se divide en cuatro partes. En la primera se sintetizan los principales elementos teóricos de la discusión alrededor del mobbing. En la segunda, se revisa la legislación sobre mobbing en Colombia. En la tercera parte, se presenta el análisis de los trabajos que han realizado las comunidades académicas del país sobre el tema. Para terminar, se argumenta sobre el poco desarrollo del tema en Colombia, el predominio de los trabajos teóricos y desde áreas no administrativas. También se encuentra que la heterogeneidad y el bajo número de los trabajos dificulta su comparación, sin embargo, ellos comparten un similar marco de referencia conceptual, empírico y legal.

\section{Palabras clave: Acoso laboral, mobbing, organizaciones, legislación colombiana, violencia en el trabajo.}

\begin{abstract}
Mobbing is recognized as a promising line of work and high impact on man, organizations, and society. In Latin America, and specifically in Colombia, little development has been identified on the topic; this is a potential weakness against to the possibilities of knowing and intervening against it, which is accepted as a growing reality throughout the world. The objective of this paper is to present the results of the review and analysis of the issue of mobbing for Colombia. The document is divided into four parts. The first summarizes the main theoretical elements of the discussion about mobbing. In the second, we review mobbing legislation in Colombia. The third part presents an analysis of the work done by academic communities in the country on the subject. Finally, we portray the little development on topic in Colombia, the predominance of theoretical works, and from different areas than administrative. It has also been found that the heterogeneity and the low number of jobs make comparisons difficult; however, they share a similar conceptual, empirical, and legal framework.
\end{abstract}

Keywords: workplace harassment, mobbing, organizations, Colombian legislation, workplace violence.

* Artículo producto de la investigación "Los Derechos Humanos en las organizaciones de Manizales: concepciones, políticas y prácticas”, financiada por la Universidad Nacional de Colombia. 


\section{Résumée}

Le mobbing se reconnait comme une ligne de travail prometteuse et à haut impact sur l'homme, les organisations et la société. En Amérique Latine et plus spécifiquement en Colombie, on identifie un faible développement de cette thématique, ce qui constitue une faiblesse face aux possibilités de connaître et d'intervenir sur celleci, qui est reconnue en tant que réalité croissante dans le monde. Ĺobjectif de cet article est celui de présenter les résultats de la révision et de l'analyse du sujet du mobbing pour la Colombie. Le document est divisé en quatre parties. Dans la première, on synthétise les principaux éléments théoriques de la discussion autour du mobbing. Dans la deuxième, on révise la législation sur le mobbing en Colombie. Dans la troisième, on présente l'analyse des travaux réalisés par les communautés académiques du pays. Pour finir, on expose une argumentation sur le pauvre développement du sujet en Colombie, la prédominance des travaux théoriques et depuis des aires non administratives. On trouve aussi que l'hétérogénéité et le faible nombre de travaux rend difficile une comparaison, cependant, ils partagent un cadre de référence conceptuel, théorique et légal, similaire.

Mots clef: Harcèlement au travail, mobbing, organisations, législation colombienne, violence au travail.

\section{Introducción}

El mobbing o acoso laboral se considera tan antiguo como el propio trabajo (Gimeno, 2004), constituye un tipo de violencia psicológica y se reconoce como una línea de trabajo promisoria y de alto impacto sobre el hombre, las organizaciones y la sociedad. En la actualidad constituye un problema prioritario que afecta la dignidad, genera desigualdad, discriminación, estigmatización y conflictos (OIT, CIE, OMS y ISP, 2002). El propósito de este artículo es presentar los resultados de la revisión y el análisis del tema del mobbing para Colombia. El documento se divide en cuatro partes. En la primera, a partir de la revisión de los autores más representativos, se sintetizan los principales elementos teóricos de la discusión alrededor del mobbing como concepto, causas, consecuencias, proceso e instrumentos de medición. En la segunda, se presenta una aproximación al mobbing desde el plano legal, allí se inicia con la mención de los países que hasta el año 2011 habían legislado sobre el asunto, para luego presentar una síntesis sobre la legislación en Colombia. En la tercera parte, se presenta el análisis de las 14 investigaciones que sobre el tema han realizado las comunidades académicas del país, clasificadas en empíricos y teóricos. En las conclusiones se argumenta sobre el avance incipiente en el tema en Colombia y se muestra cómo predominan los trabajos teóricos, desde la perspectiva de la salud ocupacional, la salud pública y el derecho, mientras que, siendo de menor número, las investigaciones empíricas operan principalmente sobre metodologías cualitativas. La heterogeneidad y el bajo número imposibilitan un trabajo comparativo profundo, pero los trabajos identificados comparten un similar marco de referencia conceptual, empírico y legal. Se retoma la recomendación de un número importante de los autores revisados con respecto a la necesidad que el Estado, los académicos, empresarios y administradores asuman, desde la naturaleza de sus roles, responsabilidades frente al tema. Conviene mencionar como principal dificultad del trabajo la escases de estudios locales y su heterogeneidad.

\section{Aproximación al concepto}

La revisión sobre el tema del mobbing muestra que el auge de las investigaciones se dio entre los años 1980 y 2000 , sobre todo en Europa, más específicamente en los países escandinavos (Suecia, Noruega y Finlandia), donde la preocupación por el hombre, su bienestar y su desarrollo, permitió importantes avances.

El término mobbing proviene del verbo inglés to mob, que traduce "regañar, atacar, maltratar, asediar", mientras que mob como sustantivo significa "muchedumbre, jauría". Desde este significado, el término expone un fenómeno de grupo (Hirigoyen, 2001, p. 70). Esta expresión fue introducida en 1963 por el etólogo y zoólogo austriaco Konrad Lorenz, para referirse a las conductas de hostigamiento de ciertos grupos de animales, que se agrupan para atacar a otro animal por el que se sienten amenazados (Piñuel y Oñate, 2002). En 1972 el psiquiatra sueco Heinemann, recuperó el término para referirse a la conducta hostil de algunos niños frente a otros en el ambiente escolar ${ }^{1}$ (Hirigoyen, 2001). En 1976, el psiquiatra estadounidense Brodsky describió el fenómeno como

[...] intentos repetidos y persistentes de un individuo de atormentar, desgastar, frustrar o conseguir una reacción de otro individuo, mediante un trato que continuamente provoca, presiona, asusta, intimida o causa la incomodidad de otro individuo en el trabajo (Einarsen, 2000, p. 382).

En la década de 1980 , el psiquiatra alemán Leymann $^{2}$ inició investigaciones sobre el tema sin conocer la obra de Brodsky. Él adoptó el término mobbing al encontrar en el trabajo conductas similares a las del mundo animal ${ }^{3}$. Para Leymann, el mobbing se caracteriza por conductas sofisticadas, sutiles y cínicas (Leymann, 1996a).

1. Fenómeno al que se ha denominado bullying.

2. Establecido en Suecia e influido por la Ley sobre condiciones de trabajo en ese país en 1976.

3. Mantuvo el término mobbing y no bullying, ante la connotación físicamente agresiva que caracteriza a este último. Bullying se utiliza para la violencia en el contexto escolar. También como equivalente a mobbing por autores de habla inglesa (Escartín et al., 2009). 
En 1996 Leymann publica el libro “Mobbing. La persecución en el trabajo ${ }^{4 "}$ ", impreso en francés y luego difundido especialmente en Europa, Estados Unidos, Australia, Sudáfrica y España (Hirigoyen, 2001) 4 .

El término mobbing es el más reconocido para referirse a la violencia psicológica en el trabajo. Pero no hay una definición aceptada por la comunidad científica y existe variedad de términos para referirse al fenómeno.

De Elena y Peña (citado por Escartín, Arrieta y Rodríguez 2009) propone clasificar las diferentes acepciones en cuatro grupos, con el fin de esclarecer el término mobbing: 1. Términos relacionados pero poco equivalentes (opresión, subrogación, comportamiento abusivo, etc.); 2. Términos que refieren a un concepto más amplio (violencia, agresión o comportamiento antisocial en el trabajo); 3. Expresiones parciales (bossing ${ }^{5}$, tiranía mezquina); y 4. Denominaciones equivalentes (terror psicológico, acoso moral, violencia psicológica en el lugar de trabajo, hostigamiento psicológico, bullying, abuso emocional en el trabajo) (p. 6).

El mobbing hace referencia a cualquier manifestación de una conducta negativa, ya sea hostil, inmoral (Leymann, 1996a), abusiva (Hirigoyen, 2001) o cruel (Piñuel, 2004), realizada de manera deliberada, continua (Piñuel, 2004), repetitiva y sistemática (Hirigoyen, 2001), por uno o más individuos de la organización, contra un único individuo (Leymann, 1996a; Piñuel, 2004), con el objetivo de humillar, desprestigiar, estresar, hostigar, ofender, excluir, interferir negativamente en sus tareas laborales (Einarsen y Hauge, 2006), maltratar, destruir psicológicamente y al final obtener su salida de la organización (Piñuel, 2004) mediante comportamientos, actitudes, gestos, palabras (Hirigoyen, 2001), escritos, actos, procedimientos ilegales o ilícitos, que atentan contra su personalidad (Piñuel, 2004), dignidad, integridad psíquica o física y que degradan el ambiente de trabajo (Hirigoyen, 2001).

Desde algunos autores, estas acciones, para poder constituir mobbing, deberían ocurrir regularmente (al menos una vez por semana) y durante un tiempo prolongado (por lo menos de seis meses) (Leymann, 1996a; Leymann y Gustafsson, 1996; Einarsen, 2000; Mikkelsen y Einarsen, 2001). Demostrar una conducta hostil no es fácil. El acoso laboral suele ser sutil, perverso y predeterminado; tiene por objetivo anular y avasallar totalmente a la víctima, en forma de “crimen perfecto" (Del Pino y Del Pino, 2007).
En muchas ocasiones el acoso laboral se da por razones de género, vulnerando el derecho a la igualdad ${ }^{6} \mathrm{y}$ a la no discriminación. Sin embargo, algunas investigaciones muestran que no hay diferencia significativa entre mujeres y hombres (Leymann, 1996a; Hoel y Cooper, 2000); lo que sí es determinante es el género del acosador (los hombres en su mayoría son acosados por hombres y las mujeres por mujeres) la razón, la estructura de la organización del trabajo, donde se conforman grupos de trabajo del mismo género (Leymann, 1996a, p. 11).

Ocurren conductas que ocasionan consecuencias importantes sobre la persona, pero que no son necesariamente mobbing. Son algunos ejemplos los incidentes aislados como roces, fricciones y tensiones que generan discrepancias (Piñuel, 2001), las advertencias airadas (Hirigoyen, 1999), el burnout ${ }^{7}$ o síndrome de estar quemado en el trabajo (Gil y Peiró, 1999), La diferencia radica en la intención de causar daño y el realizar las acciones contra la víctima de forma repetitiva y continua (Randstad, 2003) ${ }^{8}$.

El acoso sexual no es per se acoso laboral ${ }^{9}$. El objetivo del mobbing es la autoeliminación laboral; en el acoso sexual el objetivo es el sexo. El tiempo que transcurre en un tipo de acoso y el otro no es comparable. La percepción de la víctima y las consecuencias sufridas son diferentes (Gimeno, 2004). A pesar de las diferencias, pasar de un fenómeno a otro es frecuente. En muchos casos el mobbing comienza con comentarios sexistas (Hirigoyen, 2001).

\subsection{Las causas del mobbing}

Einarsen (2000) identificó tres modelos para abordar las causas del acoso psicológico: 1) en función de la personalidad de la víctima y del acosador; 2) en función de las características inherentes a las interacciones humanas en las organizaciones; y 3 ) en el clima y el ambiente organizacional específicos de una organización (p. 388).

Desde los rasgos de la personalidad de la víctima, son causas potenciales la baja autoestima, la timidez (Einarsen, 1999), la forma de actuar, las capacidades para desempeñarse en el trabajo, el perfeccionismo (Hirigoyen, 2001) y la envidia (Björkqvist, Österman y Hjelt-Bäck, 1994). Las víctimas suelen ser personas catalogadas como íntegras, triunfadoras, entusiastas, con valores morales y respetuosas de los derechos humanos

4. Los países pioneros en legislar fueron: Suecia (1992), Australia (1996), Francia (1999), Gran Bretaña (2002), Estados Unidos (2002) y Québec (2003) (Peña y Sánchez, 2006).

5. Término equivalente al tipo de acoso descendente.

6. En España la Ley $3 / 2007$, hace una referencia al acoso laboral realizado en función del género.

7. El burnout es "una respuesta al estrés laboral crónico donde el individuo desarrolla una idea de fracaso profesional (en especial en relación con las personas hacia las que trabaja)" (Gil y Peiró, 1999, p. 261).

8. Diferenciación del Instituto Sindical de Trabajo, Ambiente y Salud (ISTAS) de España.

9. En Colombia la Ley 1010 de 2006 sobre acoso laboral, incluye dentro de sus modalidades el acoso sexual, sin embargo en otros países la legislación sobre acoso laboral lo excluye. 
(Gates, 2004). Pero estos rasgos, no predisponen necesariamente a la persona a ser víctima, cualquiera puede llegar a serlo (Trujillo, Valderrabano y Hernández, 2007). Para Hirigoyen (1999) la víctima lo es por la voluntad del agresor. A partir de la designación de este, aquella se convierte en "blanco" de la violencia y en ocasiones es "señalada injustificadamente por los compañeros como cómplice de la violencia que recibe" (p. 121).

Respecto al acosador, algunos de sus comportamientos de agresión se pueden explicar por su personalidad narcisista ${ }^{10}$, consistente en una autoimagen enfermizamente sobrevalorada, percibe a los demás como fuente de amenaza, y por ende, desencadena la violencia (Piñuel, 2001). El acosador encubre sus debilidades, para defender su reputación o su posición. Esto lo lleva a denigrar de otros (Leymann, 1996a) y a desarrollar monitoreo para detectar movimientos de la víctima, que signifiquen riesgo (Piñuel, 2001), a identificar sus debilidades y dónde puede generarle más sufrimiento (Hirigoyen, 1999).

Desde las características de las interacciones humanas, se puede generar mobbing cuando existe rivalidad por obtener un puesto de trabajo, cuando en un mismo puesto están dos trabajadores y ambos creen que la compañía quiere deshacerse de alguno (Hirigoyen, 2001). También contribuye la aprobación de comportamientos hostiles del agresor por parte de los compañeros de la víctima; cuando se niega la existencia de las conductas de acoso o se permite que este suceda ${ }^{11} y$ cuando se niega ayuda a las víctimas (Luna, 2003).

Desde lo organizacional, algunos factores causantes de mobbing son: la sobrecarga de trabajo, las deficiencias en los estilos de liderazgo (Leymann, 1990; Leymann, 1996a), las deficiencias en el diseño de la tarea, el conflicto de funciones ${ }^{12}$, insatisfacción con el clima (Einarsen, 2000), la cultura organizacional (Zapf, 1999) y los problemas de comunicación interna (Einarsen, Raknes y Matthiesen, 1994). Se argumenta también, que las políticas de aumento de productividad, reducción de costos y las situaciones de conflicto sobre derechos, constituyen factores estructurales que propician el acoso psicológico en el trabajo (López, Seco y Ramírez, 2011).

\subsection{Las consecuencias del mobbing}

Se han demostrado efectos nocivos del mobbing sobre las personas, las organizaciones y la sociedad. Gran parte de los estudios indagan sobre las consecuencias individuales y organizacionales, siendo más representativos los trabajos que se centran en los efectos sobre las personas.
Entre otros efectos, el mobbing genera depresión, ansiedad, desesperación, ira (Leymann, 1990), insomnio, síntomas nerviosos, melancolía, apatía, falta de concentración, agresividad, fobia social (Björkqvist et al., 1994), estrés (Vartia, 2001), trastorno del sueño, cambios en la personalidad, síndrome de estrés pos-traumático ${ }^{13}$ (Piñuel, 2001), problemas psicológicos, trastornos psicosomáticos (Mikkelsen y Einarsen, 2002) y suicidios (Leymann, 1990).

En el entorno social y familiar, se evidencian tensiones y conflictos, retraimiento de la víctima hacia sus familiares, abandono de los amigos, estigmatización social (Piñuel, 2001), aislamiento social, inadaptación social (Leymann, 1990), entre otras consecuencias.

En las organizaciones, las principales consecuencias asociadas con el mobbing son entre otras la disminución de productividad y compromiso, absentismo por enfermedad (Hoel y Cooper, 2000), insatisfacción en el trabajo, mayor rotación de personal, intención de dejar el puesto (Quine, 1999), costos de producción más elevados, disminución de la motivación (Leymann, 1996a), bajas laborales continuas y reducción forzada de la jornada laboral (Piñuel, 2001).

\subsection{El mobbing como proceso}

El acoso suele comenzar con un cambio brusco en una relación, pasando de normal o neutra a negativa. El proceso se torna confuso para la víctima, quien empieza a sentir culpa por lo que sucede, y a asumir que la causa tiene que ver con sus capacidades o su conducta (Piñuel, 2001).

Comienza de forma superficial, difícil de percibir. Los ataques luego se intensifican, la víctima se siente acorralada y sin comprender por qué le sucede, es sometida a actos hostiles y degradantes (Hirigoyen, 1999). Los agresores ponen a los más dóciles del grupo en contra de la víctima, quien ante el silencio y la indiferencia del grupo, reacciona con hostilidad, generando un proceso circular (Hirigoyen, 2001). Se deteriora la comunicación, la víctima empieza a ser aislada, se le asignan tareas poco importantes y de poco interés. Se atacan sus capacidades profesionales y se inicia un proceso de destrucción de su autoconfianza y autoestima (Piñuel, 2001).

El proceso de victimización es sistematizado por Leymann (1990) en cuatro fases ${ }^{14}$ :

Fase 1. Incidente crítico original. El conflicto es el estado activador del acoso. Se desarrolla en un tiempo muy corto.

Fase 2. Mobbing y estigmatización. Se identifican acciones cuya finalidad es causar daño, de forma sistemática.

10. Sobre el narcisismo en el contexto organizacional, véase Rojas, W. (2003); Cruz (2002; 2003).

11. Ya que de esta forma el resto del grupo tiene menos posibilidades de sufrirlo.

12. Se ubica a dos personas en un mismo puesto, para generar rivalidad entre ellas (Hirigoyen, 2001).

13. Característico de víctimas de asaltos, catástrofes, bombardeos y violaciones (Piñuel, 2001).

14. Piñuel (2008) describe el proceso de victimización o “la espiral del mobbing” en cinco fases, incorporando una fase adicional:

la solicitud de ayuda especializada externa y de diagnóstico incorrecto. 
Fase 3. Administración de personal. Cuando los administrativos se enteran, suelen asumir prejuicios en contra de la víctima y se atribuye el problema a sus características personales. Como solución buscan liberarse de ella, se le persigue, desprestigia y culpabiliza.

Fase 4. Expulsión. La víctima intenta defenderse, por ello y las secuelas del acoso, se agrava su situación. Finalmente abandona su trabajo y busca ayuda médica y psicológica.

\subsection{Instrumentos de medición del mobbing}

La Tabla 1 muestra los principales instrumentos que se han utilizado para identificar y medir la frecuencia de las conductas constitutivas de mobbing en el lugar de trabajo.

\begin{tabular}{|c|c|}
\hline Instrumento & Autor \\
\hline $\begin{array}{l}\text { LIPT (Leymann Inventory of Psychologi- } \\
\text { cal Terrorization) }\end{array}$ & Leymann (1990) \\
\hline LIPT-6o & $\begin{array}{l}\text { González y } \\
\text { Rodríguez (2006) }\end{array}$ \\
\hline $\begin{array}{l}\text { Barómetro CISNEROS (Cuestionario } \\
\text { Individual sobre Psicoterror, Negación, } \\
\text { Estigmatización y Rechazo en Organiza- } \\
\text { ciones Sociales) }\end{array}$ & $\begin{array}{l}\text { Fidalgo y Piñuel } \\
(2004)\end{array}$ \\
\hline NAQ (Negative Acts Questionnaire) ${ }^{15}$ & $\begin{array}{l}\text { Einarsen, S. y } \\
\text { Raknes, B. (1997) }\end{array}$ \\
\hline WHS (Work Harasment Scale) & $\begin{array}{l}\text { Björkqvist, et al. } \\
\text { (1994) }\end{array}$ \\
\hline $\begin{array}{l}\text { CAPT (Cuestionario de Acoso Psicológico } \\
\text { en el Trabajo) }\end{array}$ & $\begin{array}{l}\text { Moreno, Rodrí- } \\
\text { guez, Morante y } \\
\text { Garrosa (2005) }\end{array}$ \\
\hline \multicolumn{2}{|c|}{ Fuente: Carvajal, J.G. y Dávila, C.A. (2013 } \\
\hline
\end{tabular}

El instrumento más referenciado a nivel mundial en las investigaciones consultadas es el LIPT. En España se utiliza con frecuencia el Barómetro CISNEROS y el LIPT-6o (Moreno, Rodríguez, Morante, Garrosa, Rodríguez y Díaz, 2008). En Colombia, en los documentos consultados, se ha empleado el LIPT en el estudio realizado por el Ministerio de la Protección Social [MinProtección] y la Universidad de Antioquia (2004) y el LIPT-6o en la investigación de Camargo y Puentes (2010).

EI LIPT fue el primer cuestionario diseñado para evaluar las conductas de mobbing. Consta de 45 ítems en función de las consecuencias clasificados en cinco grupos de acciones que limitan la comunicación de la víctima, limitan el contacto social, destruyen la reputación personal, desacreditan su capacidad profesional y laboral y afectan su salud. Evalúa de forma escalar la cantidad e intensidad de las acciones de acoso.

\subsection{Epidemiología del mobbing}

Según el informe presentado por la Fundación Europea para el Mejoramiento de las Condiciones de Vida y Trabajo en el año 2000, aproximadamente 12 millones (9\%) de los trabajadores europeos sufrían de acoso laboral (Randstad, 2003) ${ }^{16}$.

De acuerdo con el Barómetro CISNEROS XI, en España 2,5 millones $(13,2 \%)$ de trabajadores activos sufren de acoso laboral, de ellos, $42 \%$ presentó daños psicológicos y las víctimas más frecuentes fueron hombres. La mitad de ellas refirieron a un hombre como el acosador. El principal tipo de acoso fue el descendente (64\%), seguido del horizontal (18\%). El mando intermedio y el operativo fueron los puestos con mayor frecuencia $(21,8 \%$ y $16,5 \%)$. A la pregunta sobre la explicación del comportamiento de los acosadores, la mayoría consideró que se debía a "un desequilibrio emocional", a "la envidia, los celos y la rivalidad" y a "la presión o estrés a que está sometido". Las causas generales fueron "la envidia", "el éxito profesional", "el no ceder ante el chantaje y el reivindicar los derechos laborales". Entre los años 2006 y 2008 la tasa de mobbing creció en 43\% (Piñuel, 2008b).

\section{Legislación sobre acoso laboral en Colombia}

Antes de revisar los aspectos relacionados con la legislación colombiana se ha considerado pertinente mostrar la información coleccionada en el plano internacional (Tabla 2).

\begin{tabular}{|c|c|}
\hline País & Legislación \\
\hline Suecia & $\begin{array}{l}\text { - Norma específica sobre acoso laboral en } \\
\text { desarrollo de su Ordenanza de Medio Ambiente } \\
\text { Laboral. } \\
\text { - Ordenanza de } 14 \text { de enero de } 1993 \text { sobre medidas } \\
\text { para la prevención de la violencia y las amenazas } \\
\text { en el trabajo. } \\
\text { - Ordenanza de } 21 \text { de septiembre de } 1993 \text { sobre } \\
\text { medidas contra el acoso en el trabajo. }\end{array}$ \\
\hline Dinamarca & $\begin{array}{l}\text { - Aprobación de una Orden sobre Actividad Laboral } \\
\text { en desarrollo de su Ley de Medio Ambiente Laboral. } \\
\text { En Mayo de } 2001 \text { se estableció un acuerdo entre } \\
\text { grupos sindicales y empleadores a fin de que exista } \\
\text { una cooperación entre empresarios y represen- } \\
\text { tantes de los trabajadores para que estén presen- } \\
\text { tes en los procedimientos que se inicien por riesgo } \\
\text { psicosocial. }\end{array}$ \\
\hline Holanda & - Reformas sobre la Ley de Condiciones de Trabajo. \\
\hline Noruega & $\begin{array}{l}\text { - Reforma a la Ley relativa a la Protección de los } \\
\text { Trabajadores y Medio Ambiente Laboral. }\end{array}$ \\
\hline Francia & $\begin{array}{l}\text { - Ley } 2002-73 \text { de } 17 \text { de enero de modernización } \\
\text { social. } \\
\text { Artículo 122-54. Código de Trabajo. }\end{array}$ \\
\hline Bélgica & $\begin{array}{l}\text { - Acuerdo entre empleadores y grupos sindicales } \\
\text { para la prevención y tratamiento del estrés } 1998 .\end{array}$ \\
\hline $\begin{array}{l}\text { Gran } \\
\text { Bretaña }\end{array}$ & $\begin{array}{l}\text { - Ley de } 27 \text { de marzo de } 1997 \text { de protección de todo } \\
\text { tipo de acoso. }\end{array}$ \\
\hline
\end{tabular}

Fuente: Carvaial. I.G. v Dávila, C.A. (2013) a partir de Garzón (2011).

15. Actualizado en 2001 por Einarsen y Hoel, dando origen al NAQ-R (Hauge, Skogstad y Einarsen, 2007).

16. Los resultados de los estudios del mobbing pueden ser contradictorios, ya que las escalas medición y los sectores estudiados son diferentes (Randstad, 2003; Martínez y Medeiros, 2010). 
En América Latina el mobbing apenas comienza a tener eco en los investigadores y son pocos los países que han promulgado leyes. Hasta el momento los países que han avanzado en este tema son: Argentina (Ley № 1225 del 5 de enero de 2004), Colombia (Ley 1010 del 23 de enero del 2006), Puerto Rico (Proyecto de Ley 1008 aprobado por la Asamblea Legislativa en octubre de 2007); en Ecuador se encuentra en discusión el Proyecto de Ley que busca contrarrestar estas conductas (Fernández y Nava, 2010, p. 73); en México, aunque existen varias "vías para hacer justicia a las víctimas del mobbing" (derecho penal, civil, constitucional, administrativo, laboral y de seguridad social) no hay mecanismos legales específicos ${ }^{17}$ (Bermúdez y García, 2012, p. 326).

España es sin duda uno de los países hispanoparlantes con mayor desarrollo legislativo sobre el tema, al punto que el acoso laboral está tipificado como delito en el Código Penal (Art. 173). Esta Ley establece pena de prisión a los funcionarios públicos que atenten contra la integridad moral (Art. 175) (España, 2010).

España también cuenta con un protocolo anti-acoso ${ }^{18}$, que establece "un procedimiento de actuación ante situaciones que pudieran constituir acoso laboral" (Cap. 2), se aplica al personal de la administración pública y está integrado al Sistema de Gestión de la Prevención de Riesgos Laborales (España, 2011).

En Colombia, la Ley 1010 de $2006^{19}$, representa un intento del Estado por intervenir respecto al acoso laboral. Expedida por el Congreso de la República el 23 de enero de 2006, con el objetivo de beneficiar a todas las personas que laboran en las organizaciones donde el ambiente de trabajo es agresivo y hostil, donde se presentan situaciones que degradan la dignidad humana y sancionar a aquellas empresas que permiten el maltrato y el abuso del trabajador en el ámbito laboral (Peralta, 2006). Esta Ley busca:

[...] definir, prevenir, corregir y sancionar las diversas formas de agresión, maltrato, vejámenes, trato desconsiderado y ofensivo y en general todo ultraje a la dignidad humana que se ejercen sobre quienes realizan sus actividades económicas en el contexto de una relación laboral privada o pública (Colombia, 2006) ${ }^{20}$.

La ley señala como bienes jurídicos protegidos "el trabajo en condiciones dignas y justas, la libertad, la intimidad, la honra y la salud mental de los trabajadores, la armonía entre quienes comparten un mismo ambiente laboral y el buen ambiente en la empresa" (Colombia, 2006) ${ }^{21}$. Para efectos de esta Ley, se define acoso laboral como:

[...] toda conducta persistente y demostrable, ejercida sobre un empleado o trabajador por parte de un empleado, jefe o superior jerárquico inmediato 0 mediato, un compañero de trabajo o un subalterno, encaminada a infundir miedo, intimidación, terror y angustia, a causar perjuicio laboral, generar desmotivación en el trabajo, 0 inducir la renuncia del mismo (Colombia, 2006) ${ }^{22}$.

Desde esta Ley, el acoso laboral puede darse, principalmente, bajo las modalidades generales de maltrato laboral, persecución laboral, discriminación laboral, entorpecimiento laboral, inequidad laboral y desprotección laboral.

En el Capítulo IV de la Resolución 2646 de 2008 el Ministerio de la Protección Social en Colombia adoptó un "Protocolo para la determinación del origen de las patologías derivadas del estrés", el cual tiene por objetivo diagnosticar y calificar la "relación de causalidad entre los factores de riesgo psicosociales" y las patologías derivadas por estrés ocupacional, las cuales deberán estar incluidas en la Tabla de Enfermedades Profesionales que establece el "Decreto 1832 de 1994 o la norma que lo adiciona, modifique o sustituya" ${ }^{23}$. También se podrá acudir a lo dispuesto sobre el tema por organismos internacionales como la OIT, OMS y OPS entre otros (Colombia, 2008).

Para Garzón (2011) la Ley 1010 de 2006 no solo presenta falencias, además considera fundamental reformularla, porque no "establece mecanismos efectivos de protección” para las personas en el lugar de trabajo (p. 154). Algunos problemas de la Ley son:

[...] la falta de una definición clara que evite las denuncias temerarias, la falta de un órgano especializado en su protección (...), la falta de sanciones ejemplarizantes en caso de que se ejecute la conducta, (...) la ausencia de una perspectiva de género en su regulación, la cual es esencial por ser las mujeres las principales víctimas de acoso sexual (p. 155).

\subsection{Investigaciones sobre acoso laboral en Colombia}

Con el objetivo de explorar los estudios sobre mobbing o acoso laboral en Colombia, se realizó una revisión sistemática (desde inicios del año 2000 hasta

17. Ley general de acceso de las mujeres a una vida libre de violencia (2007), en su capítulo II regula la violencia laboral y docente contra la mujer (Garzón, 2011, p. 134).

18. Compuesto por tres partes: 1. Procedimiento de actuación. 2. Seguimiento y control. 3. Medidas de actuación y prevención del acoso. 19. Los trabajos de Hirigoyen son la base de la legislación sobre acoso laboral (Colombia, 2004).

20. Artículo $1^{\circ}$.

21. Artículo $1^{\circ}$, segundo inciso.

22. Artículo $2^{\circ}$.

23. Capítulo IV, Resolución 2646 de 2008. 
julio de 2012) en Redalyc, Scielo y Ebsco Host, a partir de categorías como mobbing, bullying, acoso laboral, acoso moral y acoso psicológico y se establecieron los siguientes límites: artículos publicados en revistas y publicaciones colombianas (empíricas y teóricas), leyes y decretos relacionados con mobbing, estudios publicados por organismos estatales, así como tesis de universidades nacionales y cuya temática fuera aplicable al contexto colombiano. Finalmente se encontraron 14 documentos ( $5 \mathrm{em}$ píricos y 9 teóricos) que se relacionaban de forma directa con las categorías planteadas. La heterogeneidad y el bajo número de artículos impiden realizar un trabajo comparativo profundo y por tal motivo se analizan por separado.

\begin{tabular}{|c|l|l|}
\hline Tabla 3. Documentos empíricos revisados \\
\hline $\mathbf{N}$ & \multicolumn{1}{|c|}{ Autores } & \multicolumn{1}{|c|}{ Títulos } \\
\hline 1 & $\begin{array}{l}\text { Ministerio de la } \\
\text { Protección } \\
\text { Social (2004). }\end{array}$ & $\begin{array}{l}\text { Formas y consecuencias de la } \\
\text { violencia en el trabajo. }\end{array}$ \\
\hline 2 & $\begin{array}{l}\text { Peralta, M. } \\
\text { (2006). }\end{array}$ & $\begin{array}{l}\text { Manifestaciones del acoso } \\
\text { laboral, mobbing y síntomas } \\
\text { asociados al estrés } \\
\text { postraumático. }\end{array}$ \\
\hline 3 & $\begin{array}{l}\text { Camargo, J.y } \\
\text { Puentes A. } \\
\text { (2010). }\end{array}$ & $\begin{array}{l}\text { Rasgos de personalidad y } \\
\text { autoestima en víctimas de acoso } \\
\text { laboral. }\end{array}$ \\
\hline 4 & $\begin{array}{l}\text { López, Seco, y } \\
\text { Ramírez (2011). }\end{array}$ & $\begin{array}{l}\text { Prácticas de acoso laboral en } \\
\text { empresas colombianas: una } \\
\text { mirada estructural e inter- } \\
\text { subjetiva. }\end{array}$ \\
\hline 5 & $\begin{array}{l}\text { Castillo, J.y } \\
\text { Cubillos, A. } \\
\text { (2012). }\end{array}$ & $\begin{array}{l}\text { La violencia en las transforma- } \\
\text { ciones de los sistemas de trabajo } \\
\text { en Colombia. }\end{array}$ \\
\hline
\end{tabular}

1. El Ministerio de la Protección Social, hoy Ministerio de Salud y Protección Social, en convenio con la Universidad de Antioquia, realizó en el año 2004 un estudio de corte cuantitativo y transversal, cuyo objetivo era "(...) encontrar la forma de reconocer las variables sociales y subjetivas del fenómeno de la violencia en el trabajo" (p. 11).

Se aplicó en los sectores de vigilancia privada, transporte de carga y de pasajeros a nivel urbano e intermunicipal, en el sector salud y finanzas, desarrollado en las ciudades de Cali, Medellín, Bogotá y Barranquilla.
El estudio mostró que el acoso laboral duplicaba la prevalencia de Europa para el año 2000. Cerca del $20 \%$ de los trabajadores encuestados estaban expuestos al acoso laboral y este afectaba en mayor medida la calidad del ambiente de trabajo, la salud y vida de los trabajadores.

Los datos mostraron igual distribución entre hombres y mujeres; mayor ocurrencia en edades entre 30 y 39 años para todos los sectores, especialmente en auxiliares operativos, con formación de bachillerato o técnica. El mayor reconocimiento de ocurrencia fue para la agresión verbal (12,8\%), 3,6\% manifestó haber sufrido amenazas, con una mayor frecuencia en mujeres $(59,4 \%)$. La agresión física, representó $1,3 \%$; dos tercios de los agredidos eran hombres.

2. El artículo de Peralta (2006) expone los principales síntomas asociados con el acoso psicológico. Consiste en tres estudios de caso, aplicando entrevistas en profundidad. Como resultado, se identifican manifestaciones de acoso psicológico y se concluye que este tipo de acoso puede generar síntomas asociados con el estrés postraumático. Finalmente, propone realizar estudios de mayor profundidad que permitan visibilizar el problema, diseñar medidas preventivas y generar estrategias de acción que repercutan en la mejora de la vida laboral y garanticen y defiendan la dignidad de las personas.

3. El trabajo de Camargo y Puentes (2010) utiliza estudio de casos. Mide y describe las conductas de acoso laboral, los rasgos de personalidad y la autoestima de cuatro mujeres entre 45 y 57 años, empleadas en el sector público que denunciaron ante una instancia legal haber sido víctimas de acoso psicológico. Como resultado se pudo demostrar la presencia de acoso psicológico vertical descendente (de jefe hacia subordinado), con diferencias en la intensidad y en la frecuencia para cada caso analizado.

4. En el documento de López, Seco y Ramírez (2011) se analizan los factores estructurales y organizativos que favorecen las prácticas de acoso psicológico en Colombia. Se revisa de forma crítica la conceptualización, sobre todo la definición que de acoso psicológico plantea la Ley 1010 de 2006 . Ellos consideran que de la forma en que está planteado el acoso psicológico, hace referencia solo a las personas como actores de las prácticas de acoso, dejando de lado a las organizaciones. En este sentido, las organizaciones como instituciones creadoras y reguladoras de normas, no reciben ninguna responsabilidad por las conductas violentas que se generan a su interior ${ }^{24}$. Se realizaron 60 entrevistas semiestructuradas, orientadas a reconstruir los procesos organizativos y 15 entrevistas

24. Piñuel (2008a) advierte sobre las dificultades para evaluar periódicamente los riesgos laborales psicosociales. Según él, “las organizaciones saben que si evalúan la violencia, en especial la violencia psicológica, se la van a encontrar 'hasta en la sopa'. Por ello es mejor no evaluar, no medir" (p. 39). 
a víctimas, en dos grupos de discusión. Asimismo se revisaron 24 expedientes de demandas y querellas sobre acoso laboral.

Castillo y Cubillos (2012), hacen una aproximación comprensiva e interpretativa del fenómeno de la violencia organizacional a partir del entorno económico, social y político del país. Se comprobó la hipótesis que los trabajadores colombianos pueden tolerar más la violencia organizacional, debido a la violencia social y política que ha permeado los diferentes espacios y contextos de interacción de las personas. Para los autores, los individuos desarrollan cierta tolerancia a la violencia y a los modelos presentes en la sociedad; esta capacidad de resistir a la violencia produce que se naturalice y se integre al contexto laboral. El estudio fue de corte cualitativo y se realizaron entrevistas semi-directivas a trabajadores que hubiese experimentando alguna forma de violencia.

En la Tabla 4 se presentan los documentos teóricos encontrados, con sus autores y títulos.

\begin{tabular}{|c|c|c|}
\hline No. & Autores & Títulos \\
\hline 1 & $\begin{array}{l}\text { Peralta, M. } \\
(2004)\end{array}$ & $\begin{array}{l}\text { El acoso laboral-mobbing- } \\
\text { perspectiva psicológica. }\end{array}$ \\
\hline 2 & Rojas, A. (2005) & El acoso o mobbing laboral. \\
\hline 3 & Giraldo, J. (2005) & $\begin{array}{l}\text { Perspectiva del acoso laboral en el } \\
\text { contexto colombiano. }\end{array}$ \\
\hline 4 & $\begin{array}{l}\text { Cardona, D. y } \\
\text { Ballesteros, M. } \\
(2005)\end{array}$ & $\begin{array}{l}\text { El acoso psicológico: riesgo laboral } \\
\text { más frecuente de lo reportado. }\end{array}$ \\
\hline 5 & Muñoz, A. (2007) & $\begin{array}{l}\text { La nueva legislación sobre acoso } \\
\text { laboral en Colombia (Ley } 1010 \text { de 2006). }\end{array}$ \\
\hline 6 & $\begin{array}{l}\text { Martínez, E.; } \\
\text { Agudelo A. y } \\
\text { Vásquez E. (2010) }\end{array}$ & $\begin{array}{l}\text { Mobbing, un aspecto a vigilar en los } \\
\text { profesionales en Colombia. }\end{array}$ \\
\hline 7 & $\begin{array}{l}\text { Parra, L. y } \\
\text { Acosta, M. (2010) }\end{array}$ & $\begin{array}{l}\text { La investigación cuantitativa del } \\
\text { acoso psicológico laboral en los } \\
\text { sectores de la educación superior y } \\
\text { de la salud. Una revisión sistemática. }\end{array}$ \\
\hline 8 & Garzón, T. (2011) & $\begin{array}{l}\text { Críticas y perspectivas de la Ley } 1010 \\
\text { de } 2006: \text { una aproximación desde la } \\
\text { definición jurídica y psicológica del } \\
\text { acoso laboral. }\end{array}$ \\
\hline 9 & Álvarez, L. (2011) & $\begin{array}{l}\text { Clima social de la organización y } \\
\text { hostigamiento psicológico. Una } \\
\text { lectura cognitiva y socio-cultural } \\
\text { desde los sistemas autoritarios. }\end{array}$ \\
\hline & & Uuente: Carvajal, J.G. y Dávila, C.A. (2013). \\
\hline
\end{tabular}

En el trabajo orientado a la comunidad de psicología, Peralta (2004) elabora recomendaciones para abordar el tema desde la academia y las organizaciones; rescata la conexión que el tema tiene con la discriminación; argumenta que el acoso laboral se debe considerar un problema nuevo que ha tenido origen en la dinámica cambiante del trabajo y las nuevas tensiones internas surgidas en el proceso de globalización. Para la academia, resalta el valor de investigar sobre el tema con énfasis en estudios empíricos cualitativos de enfoque antropológico, que provean descripciones precisas del fenómeno y permitan elaborar modelos para interpretar el fenómeno e intervenirlo.

El artículo de Rojas (2005), aborda el tema desde una perspectiva jurídico-legal. En lo jurídico revisa una sentencia de la Corte Constitucional y desde lo legal analiza la legislación laboral y el proyecto de Ley 088 de 2004, que se convertiría más tarde en la Ley 1010 de 2006. Plantea que la legislación laboral establece incipientes herramientas para contrarrestar las conductas de acoso laboral, reclama por una intervención del legislador y califica favorablemente el proyecto que se abría paso en el Congreso.

Giraldo (2005) elabora una reconstrucción del concepto de acoso laboral y califica el fenómeno como disfunción organizacional frecuente. Para Colombia revisa el Proyecto de Ley 088 de 2004, el estudio de MinProtección y propone cómo hipótesis que su ocurrencia encuentra causa en la violencia del país. Asocia la reciente atención al tema como espacio para el ejercicio de la responsabilidad social y como prioridad del Estado y las empresas. Argumenta sobre el valor de la psicología organizacional para afrontar el reto que significa el fenómeno; destaca el valor que el tema representa para esa disciplina y su contribución a la sociedad.

Cardona y Ballesteros (2005) presentan una reconstrucción del tema desde la salud ocupacional y la salud pública, considerando el estudio de MinProtección y el Proyecto de Ley 88 de 2004. Coinciden con los autores coetáneos, en que hasta el año 2005 el fenómeno, como objeto de estudio, era novedoso y se perfilaba como tendencia mundial. Proponen que el acoso es constitutivo de discriminación y debe ser abordado desde diferentes disciplinas. Encuentran como escenarios de mayor probabilidad de ocurrencia organizaciones con estructuras "poco claras" y "ambientes laborales deteriorados". Advierten sobre las bondades de una legislación específica y recomiendan que el tema deba ser atendido como un asunto de salud pública.

Muñoz (2007) presenta el resultado de evaluar la Ley 1010 de 2006, reconoce que ella representa un avance legislativo frente al acoso laboral, pero llama la atención sobre aspectos en los que la ley se muestra deficitaria, entre ellos el ámbito de aplicación referido solo a la relación laboral, la imprecisión en aspectos como los criterios de persistencia y demostrabilidad de las conductas; los atenuantes, los agravantes y los criterios de gradación. El procedimiento para atender las denuncias y sus consecuencias personales y organizativas. La discriminación del proceder entre los empleados públicos y privados ${ }^{25}$. 
Martínez, Agudelo y Vásquez (2010) abordan el acoso laboral desde la perspectiva de la salud ocupacional; documentan el impacto que el fenómeno tiene a nivel individual, organizativo y social, aspectos que justifican su inclusión en el ámbito de la salud pública y la seguridad sanitaria internacional. Asignan responsabilidad a los actores sociales desde su misma naturaleza, de la comunidad académica en la realización y difusión de investigaciones, del Estado en la formulación de políticas y planes nacionales de salud, de las organizaciones en vinculación de los resultados de investigación y las políticas públicas, y argumentan sobre la necesidad de mecanismos de cooperación para lograr sincronía entre los actores. Para el estudio del tema proponen un abordaje intersectorial y utilización de metodologías multinivel y multivariadas.

Parra y Acosta (2010) presentan el resultado de analizar 50 documentos producto de abordaje cualitativo de acoso laboral en docentes universitarios, médicos y enfermeras entre 1990 y 2009. Los hallazgos describen rasgos, actitudes y comportamientos de acosadores y acosados e identifica condiciones organizacionales e individuales propicias para tal acoso. Argumentan sobre cómo sus hallazgos coinciden con varios estudios fuente del referente teórico. Sostienen al igual que López et al. (2011), que las variables organizacionales son más fuertes como predictores del acoso que las variables personales y advierten sobre los vacíos existentes en el estudio del tema frente a los aspectos organizacionales, jurídicos y de salubridad.

Garzón (2011) se aproxima al tema del acoso laboral como riesgo psicosocial y desde su conceptualización revisa el sistema legal colombiano, lo ubica en la Constitución Política, en el derecho laboral y el derecho penal. Enfatiza en las implicaciones que tiene el acoso en la afectación de los derechos humanos fundamentales, esclarece cuales resultan vulnerados en especial el derecho a la dignidad humana. Realiza una síntesis de la legislación en Europa y Latinoamérica, para terminar con una compilación de la jurisprudencia en Colombia y un detallado análisis de la Ley 1010 de 2006 y una propuesta de modificación a su articulado. Destaca la importancia de la prevención como estrategia para reducir la ocurrencia del fenómeno y la poca disponibilidad de bibliografía e información respecto a la situación de problema en Colombia.

Álvarez (2011) hace una revisión de las perspectivas comportamental y de los rasgos y el proceso del acoso laboral, luego analiza desde la cognición social y los sistemas organizativos autoritarios estudiados por Bruner (1981). Opera específicamente sobre las interacciones grupales, las creencias y las estructuras formales de organización. Concluye que el acoso encuentra explicación en las dinámicas de poder de los grupos y por tanto no debería abordarse solamente desde la perspectiva clínica.

\section{Discusión y conclusiones}

La aproximación al tema del acoso laboral permite identificar que existen numerosos estudios sobre la violencia en Colombia ${ }^{26}$, pero desde el año 2004 se avisa sobre escases de los referidos a la violencia laboral y cómo esto dificulta el acercamiento al problema.

$\mathrm{El}$ acoso laboral apenas comienza a tener espacio entre los investigadores. Entre los años 2004 y 2012 se identifica un ligero y lento avance en los estudios. Se aprecia un crecimiento en cantidad y calidad de los trabajos y se han vinculado varias comunidades académicas a la reflexión sobre el asunto; en ellas sobresalen las áreas de la salud y el derecho, sin participación de áreas propiamente empresariales. Los avances tienen que ver en primer lugar con la descripción del fenómeno en las organizaciones de varios sectores de actividad y de varias regiones, desarrollados desde la perspectiva cualitativa ${ }^{27}$ y en segundo lugar con productos de corte teórico que revisan o analizan el tema desde la perspectiva legal, desde la salud ocupacional y la salud pública. En síntesis los trabajos son muy recientes y mayoritariamente de carácter teórico.

De los documentos sobre Colombia, 5 están publicados en revistas de psicología, 4 en revistas de salud, 3 en revistas de derecho, 1 en revista de estudios sociales y 1 en una multidisciplinar. Esto demuestra que el tema del acoso laboral ha sido de mayor interés para comunidades relacionadas con las ciencias de la salud y no con empresariales propiamente dichas ${ }^{28}$.

La heterogeneidad y el bajo número de trabajos impiden una comparación profunda, sin embargo, se encuentra que la mayoría tiene un marco de referencia común respecto a la conceptualización (causas, consecuencias y proceso). También comparten como eje legal hasta el 2006, el proyecto de Ley 088 y desde este año hasta la actualidad, su formalización en la Ley 1010 de 2006. Además, tienen como referente empírico el estudio de MinProtección (2004).

Resulta escaso el número de artículos arbitrados, la poca novedad conceptual vinculada a ellos, la escases de esfuerzos de análisis y re-contextualización de materiales e instrumentos para el diagnóstico y el bajo nivel de diagnóstico en el país, que permita co-

26. Existe documentación de violencia contra sindicalistas y acciones bélicas de multinacionales a través de grupos armados ilegales. 27. De cinco trabajos empíricos disponibles cuatro son cualitativos.

28. De los artículos revisados, solo uno se encuentra publicado en una revista de gestión empresarial. 
nocer sobre su extensión, sus causas, consecuencias y perspectivas del tratamiento.

Un grupo investigadores (Peralta, 2004; Cardona y Ballesteros, 2005; Giraldo, 2005; Rojas, 2005) acepta que ampliar la comprensión de la violencia en el trabajo está relacionado con la realización de investigaciones en el campo. Otro grupo plantea que las condiciones de violencia en Colombia pueden influir en los resultados obtenidos, marcando una diferencia frente a resultados de otros países (Giraldo, 2005; Martínez et al., 2010; Garzón, 2011; Castillo y Cubillos, 2012).

Otros destacan el valor de abordar el tema desde diferentes disciplinas y de manera interdisciplinaria (Peralta, 2004; Cardona y Ballesteros, 2005; Martínez et al., 2010); esto, arrojará resultados nuevos sobre el fenómeno y sobre sus especificidades en el país. Frente a esto, se debe decir que si bien se identifican trabajos desde diferentes disciplinas, pero no trabajos propiamente interdisciplinarios.

Como recomendación con impacto importante sobre la reducción de la ocurrencia de violencia en el trabajo, varios autores demandan de acciones individuales y conjuntas del Estado, los académicos, administradores y empresarios (Peralta, 2004; Cardona y Ballesteros, 2005; Giraldo, 2005; Rojas, 2005; Muñoz, 2007; Martínez et al., 2010; Parra y Acosta, 2010; Garzón, 2011), la argumentación que mejor resume la idea es la de Martínez, et al., (2010) cuando proponen que estos tres actores asuman responsabilidad con el problema desde su misma naturaleza: los académicos en la realización y difusión de investigaciones ${ }^{29}$; el Estado en la formulación de políticas, planes y normas; y las organizaciones en la vinculación de los resultados de investigación y políticas públicas, así como el favorecer mecanismos de cooperación para lograr sincronía con las acciones de los otros dos actores.

La iniciativa realizada por el Ministerio de la Protección Social en convenio con la Universidad de Antioquia y las organizaciones de los sectores estudiados, es un buen ejemplo de que esta cooperación es posible y los beneficios emergen para los actores individualmente considerados y para la sociedad en general.

Para finalizar, si bien, la situación de Colombia frente al tema muestra un desarrollo que se ha catalogado de leve, a nivel internacional se identifican desarrollos importantes. Para atender la demanda de esfuerzos de apropiación de referentes y de recontextualización de los instrumentos de medición, resulta valioso apoyarse en una primera fase en la experiencia y los desarrollos españoles que tienen mucho que aportar al país desde lo teórico, lo metodológico y lo legal.

En Colombia, a pesar de contar con la Ley 1010 de 2006, las conductas de acoso laboral son cotidianas en las organizaciones y vulneran los derechos.

\section{Referencias}

Álvarez, L. (2011). Clima Social de la organización y hostigamiento psicológico. Una lectura cognitiva y socio-cultural desde los sistemas autoritarios. Revista Intercontinental de Psicología y Educación, (1) (pp. 83-98).

Bermúdez, G. y García, Z. (2012). El acoso laboral y la seguridad social México-España. México D.F., México: Universidad Autónoma de México.

Björkqvist, K. Österman, K. \& Hjelt-Bäck, M. (1994). Aggression among university employees. Aggressive behavior, 20 (3) (pp. 173-184).

Camargo, J. y Puentes, A. (2010). Rasgos de personalidad y autoestima en víctimas de acoso laboral. Revista Diversitas, 6 (1) (pp. 51-64).

Cardona, D. y Ballesteros, P. (2005). El acoso psicológico: riesgo laboral más frecuente de lo reportado. Revista Facultad Nacional de Salud Pública, 23 (2) (pp. 99-106).

Castillo, J. y Cubillos, Á. (2012). La violencia en las transformaciones de los sistemas de trabajo en Colombia. Revista Facultad Nacional de Salud Pública, 14 (1) (pp. 84-97).

Colombia. Congreso de la República. (2004). Exposición de motivos, Proyecto de ley No. o88. "Por medio del cual se adoptan medidas para prevenir, corregir y sancionar el acoso laboral y otros hostigamientos en el marco de las relaciones de trabajo". Bogotá, Colombia: Congreso de la República.

Colombia. Congreso de la República. (23/01/2008). "Ley 1010 de 2006", por medio de la cual se adoptan medidas para prevenir, corregir y sancionar el acoso laboral y otros hostigamientos en el marco de las relaciones de trabajo. Diario Oficial n. 46.160 (pp. 1-10).

Colombia. Ministerio de la Protección Social. (2008). "Resolución 2646 de 2008", Por la cual se establecen disposiciones y se definen responsabilidades para la identificación, evaluación, prevención, intervención y monitoreo permanente de la exposición a factores de riesgo psicosocial en el trabajo y para la determinación del origen de las patologías causadas por el estrés ocupacional. Bogotá, Colombia: Ministerio de la Protección Social.

Cruz, F. (2002). El mundo del trabajo y las organizaciones desde la perspectiva de las prácticas inhumanas. Cuadernos de Administración, 18 (27) (pp. 14-22).

Cruz, F. (2003). La presencia de lo inhumano en la cultura y las organizaciones. En F. Cruz, O. Acktouf y R. Carvajal (Eds.), El lado inhumano de las organizaciones (pp. 15-64). Cali, Colombia: Facultad de Ciencias de la Administración, Universidad del Valle.

De-Elena, J. (2005). Los problemas de denominación en el estudio del mobbing. En J. Romay, J. Salgado, M. Romaní y D. Robla (Eds.), Psicología de las organizaciones, del trabajo y recursos humanos y de la salud. Madrid, España: Biblioteca Nueva, (pp. 699-705).

Del-Pino, R. y Del Pino, M. (2007). Hacia la ética de la omisión o el desenmascaramiento de la falsa moral en las organiza-

29. También de la academia puede demandarse la transferencia y adaptación de resultados e instrumentos de medición de otros países y contribuir al esfuerzo de coordinar los otros los actores involucrados. 
ciones: mobbing y escenarios conspiracionales. En F. Peña, P. Ravelo \& S. Sánchez (Eds.), Cuando el trabajo nos castiga. Debates sobre el mobbing en México. Barcelona, España: Ediciones Eón/UAM-Azcapotzalco/Servicio Europeo de Información sobre el Mobbing (pp. 296).

Einarsen, S.,Raknes, B. \& Matthiesen, S. (1994). Bullying and harassment at work and their relationships to work environment quality: An exploratory study. European journal of work and organizational psychology, 4 (4) (pp. 381-401).

Einarsen, S. \& Raknes, B. (1997). Harassment in the workplace and the victimization of men. Violence and victims, 12 (3) (pp. 247-263).

Einarsen, S. (1999). The nature and causes of bullying at work. International Journal of Manpower, 20 (1/2) (pp.16-27).

Einarsen, S. (2000). Harassment and Bullying at work: A review Scandianvian Approach. Agression and violent Behavior, 5 (4) (pp. 379-401).

Einarsen, S. y Hauge, L. (2006). Antecedentes y consecuencias del acoso psicológico en el trabajo: una revisión de la literatura. Revista de Psicología del Trabajo y de las Organizaciones, 22(3) (pp. 251-274).

Escartín, J., Arrieta, C. y Rodríguez, A. (2009). "Mobbing” o acoso laboral: revisión de los principales aspectos teóricometodológicos que dificultan su estudio. Actualidades en Psicología, 23(110) (pp. 1-19).

España. Jefatura del Estado. (23/06/2010). “Ley Orgánica 5/2010, de 22 de junio", por la que se modifica la Ley Orgánica 10/1995, de 23 de noviembre, del Código Penal. Agencia Estatal. Boletín Oficial del Estado n. 152 (pp. 54811-54883).

España. Ministerio de Política Territorial y Administración Pública. (01/06/2011). "Resolución de 5 de mayo de 2011", de la Secretaría de Estado para la Función Pública, por la que se aprueba y publica el Acuerdo de 6 de abril de 2011 de la Mesa General de Negociación de la Administración General del Estado sobre el Protocolo de actuación frente al acoso laboral en la Administración General del Estado. Agencia Estatal, Boletín Oficial del Estado n. 130 (pp. 53956- 53969).

Fernández, M. y Nava, Y. (2010). El mobbing o acoso moral en el trabajo y su tratamiento en Venezuela. Revista de Derecho, 33 (pp. 62-95).

Fidalgo, A. y Piñuel, I. (2004). La escala Cisneros como herramienta de valoración del mobbing. Psicothema, 16 (4) (pp. 615-624).

Garzón, L. (2011). Críticas y perspectivas de la ley 1010 de 2006: una aproximación desde la definición jurídica y psicológica del acoso laboral. Bogotá, Colombia: Facultad de Jurisprudencia, Colegio Mayor de Nuestra Señora del Rosario.

Gates, G. (2004). Bullying and mobbing (Cap. 2). Pulp \& Paper, 78 (10) (p. 31).

Gil, P. y Peiró, J (1999). Perspectivas teóricas y modelos interpretativos para el estudio del síndrome de quemarse por el trabajo. Anales de Psicología, (oo2) (pp. 261-268).

Gimeno, R. (2004). La presión laboral tendenciosa (mobbing). Tesis doctoral. Universitat de Girona. Girona, España.

Giraldo, J. (2005). Perspectiva del acoso laboral en el contexto colombiano. Diversitas, 1 (2) (pp. 205-216).

González de-Rivera, J. y Rodríguez, M. (2006). Acoso psicológico en el trabajo y psicopatologia: Un estudio con el LIPT-6o y el SCL 90-R. Revista de Psicología del Trabajo y de las Organizaciones, 22 (3) (pp. 397-412).

Hauge, L., Skogstad, A. \& Einarsen, S. (2007). Relationships between stressful work environments and bullying: Results of a large representative study. Work \& Stress, 21 (3) (pp. 220-242).

Hirigoyen, M. (1999). El acoso moral: el maltrato psicológico en la vida cotidiana. Barcelona, España: Paidos Iberica Ediciones SA.

Hirigoyen, M. (2001). El acoso moral en el trabajo: Distinguir lo verdadero de lo falso. Barcelona, España: Paidos Iberica Ediciones SA.

Hoel, H. \& Cooper, C. (2000). Destructive conflict and bullying at work. Unpublished report. Manchester, United Kingdom: University of Manchester Institute of Science and Technology (UMIST).

Leymann, H. (1990). Mobbing and psychological terror at workplaces. Violence and victims, 5 (2) (pp. 119-126).

Leymann, H. (1996a). The content and development of mobbing at work. European journal of work and organizational psychology 5(2), (pp. 165-184).

Leymann, H. (1996b). Mobbing. La persécution au travail. Francia: Seuil.

Leymann, H. y Gustafsson, A. (1996). Mobbing en el Trabajo y el Desarrollo de Trastornos por Estrés Postraumático. European journal of work and organizational psychology, 5 (2) (pp. 251-275).

López, C., Seco, E. y Ramírez, D. (2011). Prácticas de acoso laboral en empresas colombianas: una mirada estructural e inter-subjetiva. Cuadernos de Administración, 24 (43) (pp. 307-328).

Luna, A. (2003). Acoso psicologico en el trabajo (mobbing). Madrid, España: Secretaria de salud laboral.

Martínez, A. y Medeiros, L. (2010). Anatomía de una confusión: error diagnóstico de patología paranoide en víctimas de mobbing. Revista de Psiquiatría Clínica, 37 (p. 4).

Martínez, E., Agudelo, A. y Vásquez, E. (2010). Mobbing, un aspecto a vigilar en los profesionales en Colombia. Revista Gerencia y Políticas de Salud, 9 (19) (pp. 41-52).

Mikkelsen, E. \& Einarsen, E. (2001). Bullying in Danish worklife: Prevalence and health correlates. European journal of work and organizational psychology, 10 (4) (pp. 393-413).

Mikkelsen, E. \& Einarsen, S. (2002). Relationships between exposure to bullying at work and psychological and psychosomaty health complaints: the role of state negative affectivity and generalized self-efficacy. Scandinavian Journal of Psychology, 43 (5) (pp. 397-405).

Colombia. Ministerio de la Protección Social. (2004). Violencia en el trabajo. Formas y consecuencias de la violencia en el trabajo: Colombia 2004. Medellín, Colombia: Ministerio de la Protección Social y Universidad de Antioquia.

Moreno, B., Rodriguez, A., Garrosa, E. y Morante, E. (2005). Antecedentes organizacionales del acoso psicologico en el trabajo: un estudio exploratorio. Psicothema, 17 (4) (pp. 627-632).

Moreno, B., Rodríguez, A., Morante, M., Garrosa, E., Rodríguez, R. y Díaz, L. (2008). Evaluación del acoso psicológico en el trabajo: desarrollo y estudio exploratorio de una escala de medida. Universitas Psychologica, 7 (2) (pp. 335-345).

Muñoz, A. (2007/12/0o). La nueva legislación sobre acoso laboral en Colombia (Ley 1010 del 2006). Revista latinoamericana de Derecho Social, n. 5 (pp. 239-246).

OIT, CIE, OMS y ISP (2002). Directrices marco para afrontar la violencia laboral en el sector de la salud. Ginebra, Suiza: Organización Internacional del Trabajo.

Parra, L. y Acosta, M. (2010). La investigación cuantitativa del acoso psicológico laboral en los sectores de la educación 
superior y de la salud. Una revisión sistemática. Revista Entramado, 11 (pp. 158-172).

Peña, F. y Sánchez, S. (2006). El mobbing. Su contenido y significado. En V Congreso de la Asociación Mexicana de Estudios del Trabajo, en la mesa "Violencia y trabajo". Ponencia presentada en el Congreso llevado a cabo en Oaxtepec, México, del 17 al 19 de mayo del 2006. Asociación Mexicana de Estudios del Trabajo.

Peralta, M. (2004). El acoso laboral -mobbing- perspectiva psicológica. Revista de Estudios Sociales, (18) (pp. 111-122).

Peralta, M. (2006). Manifestaciones del acoso laboral, mobbing y síntomas asociados al estrés postraumático: estudio de caso. Psicología desde el Caribe, (17) (pp. 1-26).

Piñuel, I. (2001). Mobbing: Cómo sobrevivir al acoso psicológico en el trabajo. Santander, España: Editorial Sal Terrae.

Piñuel, I. (2004). Informe Cisneros V: La incidencia del mobbing o acoso psicológico en el trabajo en la administración (AEAT e IGAE): resultados del barómetro Cisneros $V$ sobre violencia en el entorno laboral. Madrid, España: Sindicato GESTHA y Asociaciones Profesionales de los Cuerpos Técnicos del Ministerio de Hacienda.

Piñuel, I. (2008a). Mobbing. Estado de la cuestión. Todo lo que siempre quiso saber y nadie explicó sobre el acoso psicológico en el trabajo. Barcelona, España: Gestión 2000.

Piñuel, I. (2008b). Barómetro Cineros XI “Liderazgo Tóxico y Mobbing en la crisis económica”. Madrid, España: Instituto de Innovación Educación Educativa y Desarrollo Directivo.
Piñuel, I. y Oñate, A. (2002). La incidencia del mobbing o acoso psicologico en el trabajo en españa: Resultados del Barometro Cisneros II sobre la violencia en el entorno laboral. Lan Harremanak, 7 (pp. 35-62).

Quine, L. (1999). Workplace bullying in NHS community trust: staff questionnaire survey. British Medical Journal, 318 (pp. 228-232).

Randstad. (2003). Informe Randstad. Calidad del Trabajo en la Europa de los Quince. El acoso laboral. Madrid, España: Instituto de Estudios Laborales (IEL), ESADE-Randstad.

Rojas, A. (2005). El acoso o “mobbing” laboral. Revista de Derecho, (24) (pp. 230-245).

Rojas, W. (2003). Modernidad e inhumanidad: Lo inhumano en la organización y en el trabajo. Cali, Colombia: Editorial Universidad del Valle.

Trujillo, M., Valderrabano, M. y Hernández, R. (2007). Mobbing: historia, causas, efectos y propuesta de un modelo para las organizaciones mexicanas. Innovar: Revista de Ciencias Administrativas y Sociales, 17 (29) (pp. 71-92).

Vartia, M. (2001). Consequences of workplace bullying with respect to the well-being of its targets and the observers of bullying. Scandinavian journal of work, environment \& health 27 (1) (pp. 63-69).

Zapf, D. (1999). Organizational, work group relate and personal causes of mobbing/bullying at work. International Journal of Manpower, 20 (1/2) (pp. 70-85).

Cuadernos de Administración / Facultad de Ciencias de la Administración / Universidad del Valle Periodicidad: semestral / ISSN impreso Nº 0120-4645- ISSN electrónico No 2256-5078 / Nombre abreviado: cuad.adm. Edición Vol. $29 \mathrm{~N}^{\circ} 49$ (enero - junio de 2013) Mobbing o acoso laboral. Revisión del tema en colombia / José G. Carvajal Orozco y Carlos A. Dávila Londoño 